INPLASY

PROTOCOL

To cite: Chen et al.

Acupuncture for internet addiction: Protocol of a systematic review and metaanalysis. Inplasy protocol 2020120099. doi:

10.37766/inplasy2020.12.0099

Received: 19 December 2020

Published: 20 December 2020

Corresponding author:

Yalin Chen

ylchenyalin@163.com

Author Affiliation:

Chengdu University of

Traditional Chinese Medicine

Support: Science Foundation of China.

Review Stage at time of this submission: The review has not yet started.

Conflicts of interest:

None.

\section{Acupuncture for internet addiction: Protocol of a systematic review and meta-analysis}

Chen, Y1; Zhang, L2; Liu, Y3; Yang, Y4; Qiu, M5; Wang, Y6; Peng, W7; Wang, J8; Hao, Q9; Tu, Y10; Li, H11; Zhu, T12.

Review question / Objective: P: internet addicts over the age of 18; O: The Internet Addiction Scale score has improved significantly compared to before treatment; I、C: improvement of internet carving, anxiety, depression; S: Randomized controlled trial.

Condition being studied: Internet addiction has become a global problem, which is characterized by excessive use of the Internet, compulsive and deleterious personal behaviors.The purpose of this systematic review is to evaluate the efficacy and safety of acupuncture in the treatment of Internet addiction (IA).

INPLASY registration number: This protocol was registered with the International Platform of Registered Systematic Review and Meta-Analysis Protocols (INPLASY) on 20 December 2020 and was last updated on 20 December 2020 (registration number INPLASY2020120099).

\section{INTRODUCTION}

Review question / Objective: P: internet addicts over the age of 18; $O$ : The Internet Addiction Scale score has improved significantly compared to before treatment; I、C: improvement of internet carving, anxiety, depression; S: Randomized controlled trial.

Condition being studied: Internet addiction has become a global problem, which is characterized by excessive use of the Internet, compulsive and deleterious personal behaviors.The purpose of this 
systematic review is to evaluate the efficacy and safety of acupuncture in the treatment of Internet addiction (IA).

\section{METHODS}

Search strategy: The following databases will be searched from the inception to 30 september 2020: the cochrane library,pubmed, Embase,Web of Science,China National Knowledge Infrastructure (CNKI), Wan - fang database,Chinese Biomedical Literature Database (CBM), Chinese Scientifific Journal Database (VIP).

Participant or population: All participants were diagnosed with IA, participants were not restricted by race and gender.

Intervention: Studies involving auricular acupuncture, scalp acupuncture, electroacupuncture,during the course of treatment, the acupuncture treatment method was not restricted, and the time and frequency of needles were kept fixed.

Comparator: The control group was treated with sham acupuncture.

Study designs to be included: The eight databases will be searched from the inception to september 2020 . The research on acupuncture and internet addiction meets the screening criteria, and citation screening, data extraction, and risk assessment of bias were performed by two independent reviewers. Cochrane Review Manager 5.3 software was used for statistical analysis.

Eligibility criteria: RCTs evaluating acupuncture treatment of IA will be included. Non-randomized controlled trial, observational research, case reports were excluded. The language of the literature was Chinese or English.

Information sources: The literature search will be identified by eight databases, the Cochrane library,Pubmed, Embase,Web of Science, and four Chinese databases including WANFANG, CNKI, CBM, and VIP database,We searched these databases from their inception at september 2020. Literature language will be excluded except English and Chinese. if necessary, we will contact the author of the original article for more information or clarification. if accurate data cannot be obtained. the available data will be analyzed.

Main outcome(s): The primary efficacy endpoint was the improvement of internet craving (checked by IAT scale), self control(SCS scale), anxiety(SAS scale) and depression (SDS scale). The secondary efficacy endpoint include the average time spent on the internet (hours/day), impulsivity (BIS-11 scale), and assessment of scales related to quality of life.The results of dichotomous data were expressed as a risk ratio with $95 \% \mathrm{Cl}$, for continuous data, we used mean difference or standardized mean difference to evaluate the effect of treatment with $95 \%$ Cl.

Quality assessment / Risk of bias analysis: Two reviewers will assess the risk of bias using the cochrane collaboration tool. The content of the assessment includes: generation of random sequence (selection bias), allocation concealment (selection bias), blinding participants and personnel (performance bias), results blinding of evaluators (detection bias), incomplete result data(attrition bias), selective reporting(reporting bias)and other sources of bias,the risk of bias will be rated as "low risk", "high risk" or "unclear risk" in each area.

Strategy of data synthesis: The literature search will be identified by eight databases, the cochrane library, pubmed, embase, web of science, and four chinese databases including WANFANG, CNKI, CBM, and VIP database, we searched these databases from their inception at september 2020. Literature language will be excluded except English and Chinese.

Subgroup analysis: We will conduct subgroup analysis to detect the sources of heterogeneity, including general information (age,sex), type of acupuncture , 
course of treatment, type of control, results measurement.

Sensibility analysis: We conduct sensitivity analysis to screen out high-risk bias and ensure the robustness of the results, based on the missing data, sample size and research characteristics, we will re-analyze and compare the differences with the original data to evaluate the robustness.

Country(ies) involved: China.

Keywords: internet addiction, acupuncture, systematic review.

Contributions of each author:

Author 1 - Yalin Chen.

Email: ylchenyalin@163.com

Author 2 - Lingrui Zhang.

Email: 657116036@qq.com

Author 3 - Yan Liu.

Author 4 - Yan Yang.

Author 5 - Mimi Qiu.

Author 6 - Yang Wang.

Author 7 - Wei Peng.

Author 8 - Jun Wang.

Author 9 - Qinhong Hao.

Author 10 - Yang Tu.

Author 11 - Hui Li.

Author 12 - Tianmin Zhu. 